\title{
Modulares Projektportfoliomanagement - Eine Vision zur Beherrschung komplexer Projektlandschaften
}

Thorsten Lammers ${ }^{1}$; Henning Skirde ${ }^{2}$; Matthias Guertler ${ }^{1}$

${ }^{1}$ University of Technology Sydney, 15 Broadway, Ultimo NSW 2007, Australien, https://www.uts.edu.au

${ }^{2} I D$-Consult GmbH, Rupert-Mayer-Str. 46, 81379 Muenchen, Deutschland, https://www.id-consult.com

thorsten.lammers@uts.edu.au, henning.skirde@id-consult.com, matthias.guertler@uts.edu.au 


\section{Projektportfoliomanagement - Eine Herausforderung in Zeiten komplexer Projektlandschaften}

In Zeiten stetig zunehmender Globalisierung und des digitalen Wandels operieren Unternehmen mehr denn je in einem hochgradig dynamischen und unsicheren Umfeld. Diese Volatilität wirkt sich sowohl auf die Strategiedefinition aus, als auch darauf, wie unternehmensinterne und -externe Projekte gestaltet und durchgeführt werden, um flexibel auf Veränderungen reagieren zu können (Sarkar, 2016).

VUCA, als Akronym für die englischen Begriffe ,volatility, uncertainty, complexity and ambiguity" (deutsch Unbeständigkeit, Unsicherheit, Komplexität und Mehrdeutigkeit) fasst diesen Trend $\mathrm{zu}$ schwieriger werdenden Rahmenbedingungen der Unternehmensführung und des Projektmanagements treffend zusammen (Mack et al., 2016). Die spezifischen Ursachen hierzu sind zum Teil externen Ursprungs wie veränderte Kundenwünsche und technologischer Fortschritt, internen Ursprungs wie Strategiewechsel, oder gar projektspezifischen Ursprungs wie Projektverzögerungen (Martinsuo et al., 2014; Martinsuo and Killen, 2014).

Da traditionelle Ansätze zum Projekt- und Prozessmanagement aufgrund ihrer Inflexibilität in diesem Kontext schnell an ihre Grenzen stoßen, sind in den letzten Jahren vermehrt agilere Ansätze entwickelt worden. Beispielhaft seien hier „Scrum“ und „Lean Start-ups“ genannt, die eine kosteneffiziente, flexible und schnelle Anpassung bestehender, für gewöhnlich einzelner Projekte und Unternehmen ermöglichen (Darrin and Devereux, 2017; Parvan et al., 2015). Jedoch treten Projekte in Unternehmen in der Regel nicht isoliert, sondern als Bestandteil eines mehr oder weniger großen Projektportfolios auf, welches verschiedene parallel-laufende, potentielle und teilweise konkurrierende Projekte beinhalten kann.

Bei ihrer erfolgreichen Handhabung unterstützt das sogenannte Projektportfoliomanagement (PPM). Dieses kann definiert werden als Managementaktivität zur Planung und Steuerung aller Projekte einer Organisation. „Dazu gehören alle Aufgaben, welche für das Priorisieren, das Koordinieren, das Kontrollieren und das Unterstützen der anstehenden und laufenden Projekte und der notwendigen Ressourcen aus Projektportfoliosicht notwendig sind." (Bruno, 2005). Es stellt damit eine Abstrahierung vom Programmmanagement dar. Während beim Programmmanagement die Integration und das Management eine Gruppe zueinander in Beziehung stehender Projekte mit der Intention der Erreichung übergreifender Vorteile im 
Vordergrund steht, beinhaltet das PPM darüber hinaus auch die Herausforderung, strategische Vorteile durch die Koordination inhaltlich unabhängiger Projekte zu realisieren, die auf den gleichen Ressourcenpool zugreifen (Lycett et al., 2004). Man könnte sagen, dass sich Programmmanagement darauf bezieht "Projekte richtig durchzuführen", während es beim PPM auch darum geht, „die richtigen Projekte durchzuführen“ (Reyck et al., 2005).

Bestehende PPM Ansätze haben gewöhnlicherweise einen recht groben Betrachtungswinkel mit einem Projekt als kleinste Einheit und einem Fokus auf die strategische Auswahl geeigneter Projekte (Archer and Ghasemzadeh, 1999). Eine operative Betrachtung von Projekten findet in der Regel nicht statt. Diese Sichtweise kann jedoch die Wiederverwertbarkeit von Zwischenergebnissen für den Fall, dass Projekte unvorhersehbar gestoppt werden müssen, negativ beeinflussen. Gleichzeitig wird hierdurch die Identifikation von Abhängigkeiten zwischen parallel laufenden Projekten erschwert (Blichfeldt and Eskerod, 2008). Dies trifft insbesondere auf das Management von Projektportfolios im Supply Chain Management und externen Kollaborationsprojekten wie Open Innovation mit unterschiedlichsten Partnern zu. Hier kommen neben den oben erwähnten unternehmensinternen Herausforderungen noch weitere Probleme hinzu, die sich durch die Notwendigkeit der unternehmensübergreifenden (oft auch länderübergreifenden) Kommunikation und Komplexitätsoptimierung ergeben.

Es ergibt sich hierdurch folgende Situation und Herausforderung: während einerseits PPM-Ansätze gut zum strategischen Management größerer Projektlandschaften geeignet sind, besitzen sie Schwächen beim operativen Management einzelner Projekte, insbesondere bei der Reaktion auf unvorhergesehene Ereignisse. Andererseits besitzen agile Projektmanagementansätze gerade hier ihre Stärken, eignen sich jedoch nicht zum Management von Projektlandschaften. Die resultierende Frage und der Leitgedanke für diesen Beitrag ist: wie lassen sich die Stärken klassischer PPMmit denen agiler Ansätze zu einem agilen PPM-Ansatz kombinieren?

Ein derartiges agiles PPM müsste in der Lage sein komplexe Projektlandschaften effizient zu strukturieren, Abhängigkeiten zwischen Projekten zu berücksichtigen und zu reduzieren, die Nutzbarkeit von Zwischenergebnissen zu erhöhen und Projekte flexibel anpassen zu können. Diese Problembeschreibung weist eine starke Ähnlichkeit zu modularen Produktarchitekturen im Bereich des Produktionsmananegement auf, beispielsweise im Kontext von Design-forAdaptability. Produktmodularisierungsansätze ermöglichen diverse Vorteile, wie Beispiele wie der modulare Quer- und Längsbaukasten bei Volkswagen belegen 
(Schuh et al., 2013). Diese beinhalten unter anderem einen höheren Gleichteileanteil, reduzierte Entwicklungskosten durch standardisierte Komponenten und Schnittstellen, hoher Wiederverwendungsgrad von Designs, Kosteneinsparungen durch resultierende Skaleneffekte, reduzierte Abhängigkeiten zwischen Komponenten und Berücksichtigung verschiedener Modultreiber, wie Fertigbarkeit oder Recyclingfähigkeit (Blees et al., 2010).

Bestärkt durch erfolgreiche bereits existierende Übertragungen von Produktmodularisierungsansätzen auf andere Bereiche wie zum Beispiel Liefernetzwerke (Behnke, 2016), widmet sich der vorliegende Beitrag der Frage, ob und in welcher Form sich Produktmodularisierungsansätze in das Feld des PPMs übertragen und nutzen lassen. Nach einem kurzen Überblick über Produktmodularisierungsansätze in Unterkapitel 2 werden hierzu in Unterkapitel 3 Gemeinsamkeiten und Unterschiede zwischen Produkt- und Projektportfolien analysiert sowie entsprechende Anforderungen an ein sogenanntes „Modulares Projektportfoliomanagement (MPPM)“ abgeleitet. Wie ein derartiger MPPM-Ansatz aussehen könnte, wird in Unterkapitel 4 dargestellt. 


\section{Wie modulare Produktarchitekturen in der Produktion geholfen haben}

Im Umfeld produzierender Unternehmen haben in den letzten Jahren tiefgreifend Veränderungen stattgefunden. Globalisierungsgetrieben kann eine steigende Individualität von Kundenwünschen gepaart mit einem international stärker werdenden Wettbewerb beobachtet werden. Die Lösung dieses Zielkonfliktes der Bereitstellung erhöhter Produktvielfalt bei gleichzeitigem Kostendruck ist zum strategischen Erfolgsfaktor geworden (Koppenhagen, 2004). Größere Produktvarietät, kürzere Innovations- und Produktlebenszyklen, niedrigere Stückkosten und höhere Produktqualität sind die Zielgrößen erfolgreicher Unternehmen (Schuh, 2005). Diesem Zielkonflikt im Bereich der Produktion und der Herausforderung steigender Komplexität und Interdependenzen von Produkten und Produktfamilien wurde bereits in den 90er Jahren durch die Einführung modularer Produktarchitekturen begegnet (Ulrich, 1995). Auch wenn die Kalkulation möglicher Einsparpotenziale schwierig und Gegenstand aktueller Forschungsvorhaben ist, sind Erfolge nachträglich gut ersichtlich (Kersten et al., 2009; Skirde et al., 2016). Hierdurch konnte Volkswagen zum Beispiel bereits 1999 Einsparungen von 1,7 Milliarden USD im Bereich der Produktentwicklung und Herstellung realisieren (Bremner, 1999). Kaum überraschend also, dass die Entwicklung und Implementierung von Methoden zur Produktmodularisierung ein lukrativer Markt geworden ist (Golfmann and Lammers, 2015).

Speziell Beispiele aus der Automobilbranche zeigen das Potential und den Mehrwert modularer Produktarchitekturen, wie beispielsweise des Modularen Längs- und Querbaukastens bei Volkswagen. Im Falle des Modularen Querbaukastens konnte beispielsweise die Anzahl der Motor- und Getriebevarianten bis 2013 um 88 Prozent reduziert werden. Zudem erlaubt die hohe Anzahl an Gleichteilen die Produktion verschiedener Modellvarianten auf einer Fertigungslinie und eine resultierende höhere Fertigungsflexibilität (Schuh et al., 2013; Skirde, 2015).

Jedoch gilt es zu beachten, dass das Ziel nicht immer eine Maximierung des Modularisierungsgrades ist. Stattdessen hängt der ideale Modularisierungsgrad vom jeweiligen Produkt- und Unternehmenskontext sowie verschiedenen Modultreibern ab, die situationsspezifisch gewichtet und abgestimmt werden müssen, wie bspw. Fertigbarkeit, Wartbarkeit oder Recyclingfähigkeit (Blees et al., 2010; Kersten et al., 2013). Da Produktentwicklungen in der Regel nicht auf der ,grünen Wiese“ stattfinden sondern im Kontext komplexer oft über Jahre gewachsener Produktarchitekturen, ist es wichtig, diese kritisch zu analysieren, um den möglichen Handlungsraum zu bestimmen (Herberg, 2017). Wie am 
Beispiel von Design-for-Adaptability beschrieben, sollten verschiedene Modultreiber, ihre mögliche Entwicklung und Einfluss auf ein technisches System ermittelt werden, um die Wahrscheinlichkeit und Wirtschaftlichkeit verschiedener alternativer Modularisierungsszenarien sowie des resultierenden idealen Modularisierungsgrades bestimmen zu können (Schrieverhoff, 2015). 


\section{3 Überlegungen zur Übertragung modularer Ansätze auf den PPM Kontext}

Der Erfolg von Modularisierungsansätzen im Produktionsbereich hat $\mathrm{zu}$ Bestrebungen geführt, den konzeptionellen Ansatz zur Optimierung auf andere Bereiche zu transferieren. Hierbei seien insbesondere die Bereiche Logistik, Business Modelling und Dienstleistungen erwähnt (Bask et al., 2011; Mayer, 2007; Pekkarinen and Ulkuniemi, 2008) sowie eine parallele systematisch abgestimmte Entwicklung von Produktarchitekturen und Lieferantennetzwerken (Behnke, 2016).

\subsection{Gegenüberstellung von Produktentwicklungs- und Projektportfoliomanagementsystemen}

Um sicherzustellen, dass sich Produktmodularisierungsansätze gewinnbringend auf den Bereich des PPM übertragen und anwenden lassen, werfen wir einen kritischen Blick auf strukturelle Unterschiede und Gemeinsamkeiten zwischen Produkt- und Projektportfolien. Abbildung 1 bietet einen Überblick über die vier Betrachtungsebenen der systematischen Gegenüberstellung von Produktentwicklung und PPM sowie einer neutralen Systemtheoriedimension. 


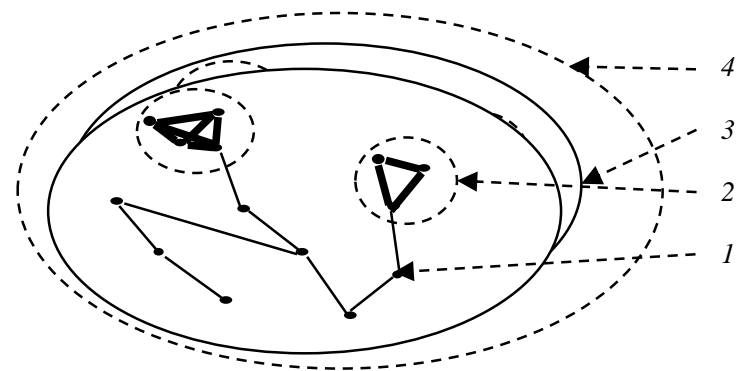

\begin{tabular}{|c|c|c|c|}
\hline Produktentwicklung & Systemtheorie & $\underline{\text { PPM }}$ & \\
\hline-----------1 & ------ & ------ & --1 \\
\hline Produktarchitektur & Transfer & $\Rightarrow$ Projektstruktur & Aggregationsebene \\
\hline Produktprogramm & Supersystem & Projektportfolio & 4 \\
\hline Produkt & System & Projekt & 3 \\
\hline Produktmodul & Modul & Arbeitspaket & 2 \\
\hline Komponente & Element & Aufgabe & 1 \\
\hline
\end{tabular}

Abbildung 1: Systemtheorie als Transferhilfe

Die identifizierten Betrachtungsebenen sind mit aufsteigendem Aggregationslevel Komponente, Produktmodul, Produkt und Produktprogramm. Eine Entsprechung lässt sich über die Systemtheorie identifizieren. In der Systemtheorie besteht ein System im Allgemeinen aus Elementen, Verbindungen und definierten Systemgrenzen (Sedlacek, 2010). Systeme können aus Submodulen bestehen, unter denen stark voneinander abhängige Elemente zusammengefasst sind. Sie sind auch oft Teil von übergreifenden Supersystemen (Haskins, 2006). Diese verallgemeinerte Betrachtung ermöglicht die Identifizierung von Analogien $\mathrm{zu}$ den Betrachtungsebenen der Produktmodularisierung im Bereich des PPM, wo dementsprechend von Aufgaben, Arbeitspaketen, Projekten und Projektportfolios die Rede ist. Tabelle 1 zeigt einen Ausschnitt einer systematischen Gegenüberstellung von Produktentwicklungs- und PPM-Systemen. 


\subsubsection{Aggregationsebene 1: "Element"}

Als Element wird der kleinste Bestandteil eines Systems bezeichnet, der zu Analysezwecken nicht weiter aufteilbar ist (Lindemann et al., 2009). In der Produktentwicklung wäre dies eine physische Komponente, wie beispielsweise eine Schraube. Diese besitzt unter anderem spezifische Eigenschaften, wie Gewicht und Größe, und Funktionalität. Das PPM-Äquivalent wäre eine Aufgabe/Aktivität innerhalb eines Projektes mit Attributen wie Arbeitsaufwand und Zielstellung/Arbeitsumfang.

\subsubsection{Aggregationsebene 2: "Modul"}

Als Modul wird im Rahmen der Systemtheorie eine Gruppe von Elementen bezeichnet, die zum Aufbau einer komplexeren Struktur genutzt werden kann (Persson and Åhlström, 2013). Idealerweise besteht diese Struktur aus Elementen, die untereinander starke Beziehungen (z. B. Information, Energie oder Material) und nach außen hin lediglich wenige, standardisierte Beziehungen aufweisen. In der Produktentwicklung werden Komponenten zu Modulen zusammengefasst, um zusammen die gleiche Funktionalität zu bedienen (z.B. eine Computertastatur als Modul des Systems „Computer“ zur Erfüllung der Funktion „Informationseingabe“). Die Entsprechung aus PPM-Sicht ist ein Arbeitspaket, welches aus Aktivitäten besteht, die zusammen das gleiche Ziel verfolgen. Beziehungen zwischen Aktivitäten können normalerweise als Abhängigkeiten verstanden werden, wie bspw. Vorbedingungen oder Abstimmungsprozesse.

\subsubsection{Aggregationsebene 3: "System"}

Ein System besteht sowohl aus in Beziehung stehenden als auch aus unabhängigen Elementen und Modulen, die organisiert und strukturiert sind (Lindemann et al., 2009). In der Produktentwicklung wird dies durch ein einzelnes Produkt repräsentiert, das sich aus Komponenten und Produktmodulen zusammensetzt. Im PPM kann ein einzelnes Projekt bestehend aus Arbeitspaketen als Analogie gesehen werden. Exemplarische Attribute sind Produktinnovationszyklen / Veränderungen des Projektumfangs, Produktabmessungen / Projektdauer und Modulverknüpfungen / Arbeitspaketabhängigkeiten.

\subsubsection{Aggregationsebene 4: "Supersystem"}

Als höchste Aggregationsebene im Rahmen dieser Analogie kann das „Supersystem“ als strukturierte Sammlung einzelner Systeme bezeichnet werden. Durch die Ausnutzung von Synergieeffekten können Ergebnisse erzielt werden, die durch alleinige Nutzung der Einzelsysteme nicht möglich wären 
(Haskins, 2006). Auf dieser Ebene ist es nun möglich, Module mit standardisierten Schnittstellen nach außen und klar definierten Funktionen zwischen einzelnen Systemen $\mathrm{zu}$ teilen oder $\mathrm{zu}$ ersetzen. In der Produktentwicklung findet sich eine Entsprechung im Produktprogramm oder einer Produktplattform, die verschiedene verwandte Produkte beinhaltet. Im Rahmen des PPM kann das aus Einzelprojekten bestehende Projektportfolio als Analogie verstanden werden.

Zusammengefasst ist festzuhalten, dass sich eine strukturelle Analogie zwischen modularen Produktarchitekturen auf der einen und Projektportfolios auf der anderen Seite herleiten lässt. Dies stellt eine wichtige Voraussetzung für einen Methodentransfer dar.

Tabelle 1: Exemplarische Gegenüberstellung von Produkt- und Projektportfolien

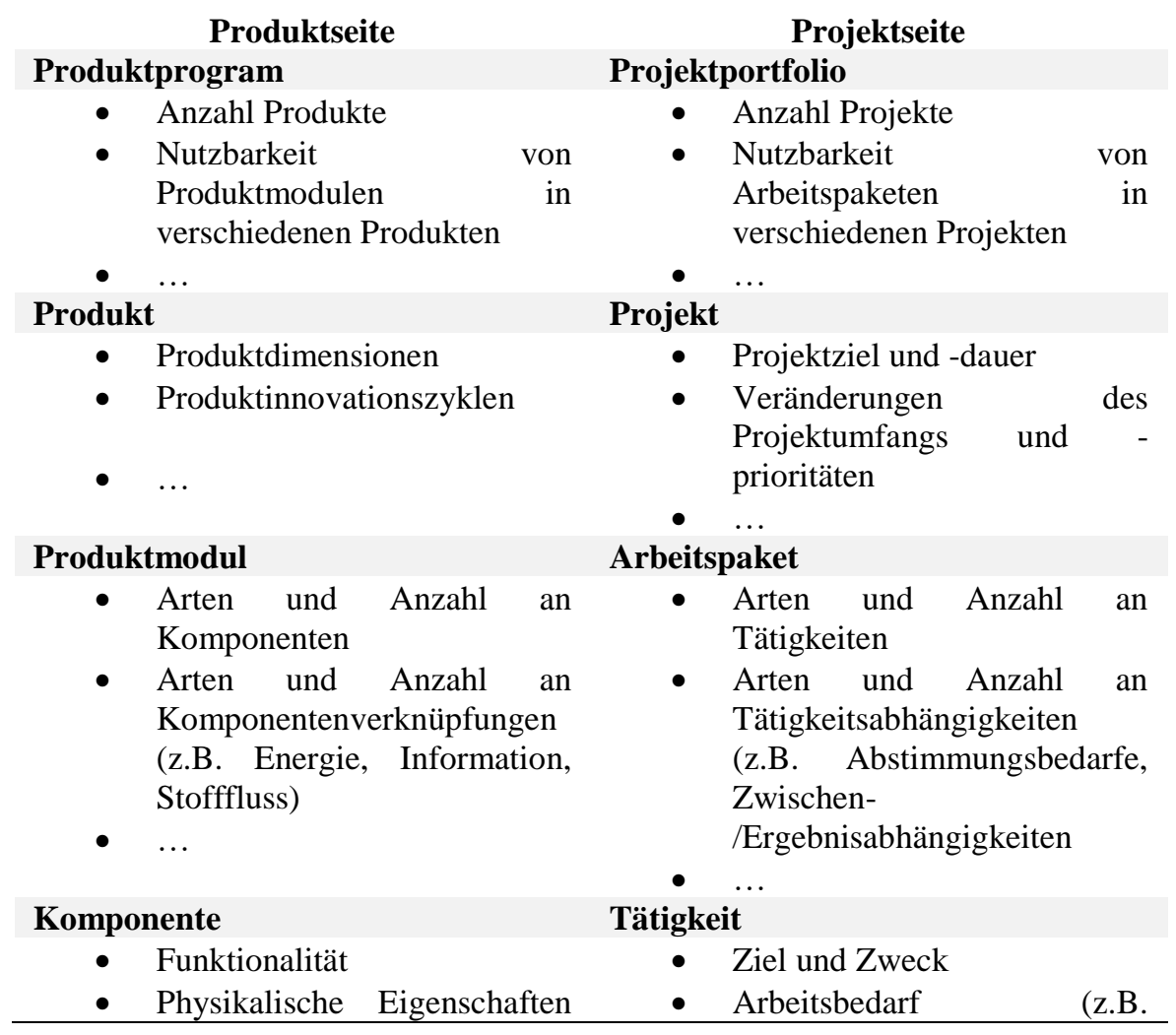


(z.B. Größe, Gewicht)

- Schnittstellenarten und anzahl (zu Komponenten)
Personenstunden)

- Schnittstellenarten und anzahl (zu Tätigkeiten)

\subsection{Notwendige Eigenschaften eines modularen Projektportfolio- managementansatzes}

In den letzten Jahren wurden zahlreiche Anstrengungen unternommen, das PPM durch die Erstellung von Standards, Modellen, formalen Bewertungskriterien und Richtlinien $\mathrm{zu}$ formalisieren (Unger et al., 2012). Formalisierte PPMModelle haben jedoch immer noch Schwierigkeiten, umfassende und angemessene Lösungen für die Projektauswahl, die Ressourcenzuteilung und den Umgang mit dynamischen Projektumgebungen bereitzustellen (Martinsuo, 2013). Speziell das Zusammenwirken und die Dynamik verschiedener Projekte und Projektaktivitäten haben sich als zentrale Komplexitätstreiber hinsichtlich der Konfiguration von Projektportfolios herausgestellt. Dieser hohe Grad an Komplexität und gegenseitigen Abhängigkeiten zwischen einzelnen Projekten stellen Entscheidungsträger beim Management dieser Portfolios vor besondere Herausforderungen. Hieraus resultiert ein allgemeiner Bedarf an verbesserten Methoden für ein verbessertes Verständnis und Verwaltung der Abhängigkeiten von Projekten. Diese bilden die Grundlage für die Identifizierung und Planung von Projekten (Killen and Kjaer, 2012).

Aufgrund des Zusammenspiels verschiedener Projekte mit individueller als auch voneinander abhängiger Unsicherheit, Dynamik und Komplexität ist es nicht zielführend, lediglich Projektmanagementansätze für PPM hochzuskalieren (Killen and Kjaer, 2012). Stattdessen werden neue eigenständige und strukturierte Ansätze benötigt, die an den jeweiligen Anwendungskontext angepasst werden können (Killen and Kjaer, 2012; Levine, 2005; Parvan et al., 2015). Traditionelle PPM-Ansätze konzentrieren sich in der Regel auf die Auswahl von Projekten auf strategischer Ebene, um den Wert des gesamten Projektportfolios zu optimieren (Archer and Ghasemzadeh, 1999). Für ein agileres PPM ist dies jedoch unzureichend (Kaiser et al., 2015). Trotz des damit verbundenen Aufwands, betonen Blichfeldt and Eskerod (2008) hierbei die Notwendigkeit einer detaillierteren Betrachtung von Projekten auf dem Level einzelner Aktivitäten. Dies bedeutet, ein agiler PPM-Ansatz sollte eine kombinierte strategische und operative Perspektive beinhalten (Patanakul, 2015). Hierbei berücksichtigt die strategische Perspektive die übergeordnete Ausrichtung der Portfolios auf die Unternehmensstrategie und die Anpassungsfähigkeit an interne und externe Änderungen. Die operative 
Perspektive gewährleistet einen systematischen Projektüberblick für verschiedene Stakeholder, Transparenz bei Entscheidungsfindungen und die Prognose von Entscheidungsergebnissen.

Um dies zu gewährleisten, muss agiles PPM sowohl an die jeweilige Unternehmensstrategie als auch die an die jeweiligen Organisationsstrukturen und -prozesse angepasst werden (Kaiser et al., 2015). Dies beinhaltet beispielsweise transparente Bewertungskriterien für eine systematische Projektpriorisierung wie Projektkosten, Zeitpläne und notwendige Ressourcen (Kaiser et al., 2015; Killen and Kjaer, 2012). Eine wesentliche Voraussetzung für ein erfolgreiches agiles PPM ist die Berücksichtigung von Projektabhängigkeiten (Killen and Kjaer, 2012; Rungi, 2010). Diese können unterteilt werden in finanzielle Abhängigkeiten (gemeinsame Finanzierungsquellen), Ressourcenabhängigkeiten (gemeinsame Infrastruktur und Ressourcen), Marktoder Nutzenabhängigkeiten (komplementäre oder wettbewerbliche Auswirkungen), Ergebnisabhängigkeiten (Verwendung anderer Projekte) und Lerneffekte (Verwendung der Erkenntnisse aus anderen Projekten) (Killen and Kjaer, 2012; Verma and Sinha, 2002). Neben der Überwachung des Verlaufs einzelner Projekte ist es daher wichtig, diese Abhängigkeiten und resultierenden Wechselwirkungen zwischen Projekten zu berücksichtigen, um ein erfolgreichen Projektcontrolling und Risikomanagement zu gewährleisten (Levine, 2005; Parvan et al., 2015). Hierbei sollten nicht nur Abhängigkeiten zwischen laufenden Projekten sondern auch Abhängigkeiten zu potenziellen Projekten in der Projektpipeline berücksichtigt werden (Levine, 2005; Rungi, 2010). Ein weiterer wichtiger Erfolgsfaktor ist zudem die Berücksichtigung und Einbindung verschiedener relevanter Stakeholder wie Projektmanager und Projektsponsoren des mittleren und oberen Managements. Tabelle 1 bietet fasst einige der wichtigsten Anforderungen an einen agilen PPM-Ansatz zusammen, die bei der Entwicklung einer modularen PPM Methodik berücksichtigt werden sollten.

Tabelle 2: Anforderungen an einen agilen Projektportfoliomanagementansatz

\begin{tabular}{l|l} 
Kategorie & Anforderung \\
\hline Agilität und & Anpassbarkeit and verschiedene Anwendungskontexte \\
\cline { 2 - 2 } \begin{tabular}{l} 
Flexibilität \\
\cline { 2 - 2 } $\begin{array}{l}\text { Handhabung } \\
\text { von }\end{array}$
\end{tabular} & $\begin{array}{l}\text { Effiziente Adaptierbarkeit der Projektlandschaft an interne } \\
\text { Komplerne Veränderungen }\end{array}$ \\
\cline { 2 - 2 } Komplexität & $\begin{array}{l}\text { Kesten, Projektdauer und notwendiger Ressourcen } \\
\text { Ressourcen, Markt, Projektergebnisse, Lerneffekte) }\end{array}$
\end{tabular}




\begin{tabular}{l|l}
\hline \multirow{2}{*}{$\begin{array}{l}\text { Strukturierter } \\
\text { Ansatz }\end{array}$} & Strukturierter und systematischer Ansatz und Vorgehen \\
\cline { 2 - 2 } & $\begin{array}{l}\text { Anpassbarkeit und Verknüpfbarkeit mit den } \\
\text { Organisationsstrukturen und -prozessen des Unternehmens }\end{array}$ \\
\hline \multirow{2}{*}{ Transparenz } & Berücksichtigung und Erfassung einzelner Projektaktivitäten \\
\cline { 2 - 2 } & $\begin{array}{l}\text { Unterstützung eines umfassenden Projektüberblicks und } \\
\text { transparenten Entscheidungsprozesses }\end{array}$
\end{tabular}




\section{Eine Vision zum Aufbau modularer Projektportfolios für Supply Chain Management- Projekte}

\subsection{Einführung des Fallbeispiels}

In den übergeordneten Kontext des modularen Projekt Portfolio Managements (MPPM) soll in diesem Kapitel ein spezifischer Anwendungsfall zunächst entwickelt und dann eingeordnet werden. Eine solche Betrachtung eines Supply Chain Management (SCM) Projektes stellt exemplarisch ein einzelnes System innerhalb des MPPM dar. Das einzelne Projekt setzt sich wiederum wird aus typischen Bausteinen wie Arbeitspaketen und Tätigkeiten zusammen. Die anwendungsorientierte Ausgestaltung unter Nutzung der etablierten Modularisierungssoftware „METUS“ soll dabei im Vordergrund stehen.

\subsection{Exemplarische Anwendung auf ein Supply Chain Management-Projekt}

\subsubsection{METUS Ansatz und Software}

METUS ist ein etabliertes Modularisierungs-Werkzeug, das eine lösungsneutrale Modellierung erlaubt, als Software für Datenhandling und Visualisierungen zur Verfügung steht und auf unterschiedliche Anwendungsfälle adaptiert werden kann. Seinen Ursprung hat METUS im Kontext der deutschen Automobilindustrie (Göpfert, 2009) und zielt vor allem auf die Strukturierung und systematische Verbesserung von Systementwicklungsprozessen ab. Ein zentraler Aspekt ist das Ableiten von Modulen, basierend auf einer funktionalen Betrachtungsweise (vgl. Abbildung 2).

Funktionsstruktur

Produktstruktur

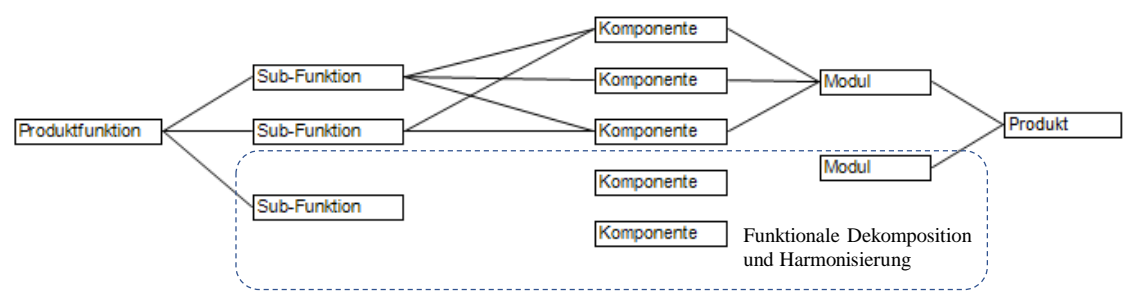

Abbildung 2: Produktarchitektur mit METUS (Göpfert, 2009) 
Gemäß Göpfert and Tretow (2013) folgt der METUS Ansatz einer vierstufigen Modularisierungsabfolge, wie im Folgenden beschrieben:

1. Klären der Ziele des Modularisierungsprojektes und identifizieren der Produktanforderungen basierend auf Kundenbedürfnissen und weiteren marktseitigen Anforderungen.

2. Entwicklung einer lösungsneutralen Funktionsstruktur zur hierarchischen Dekomposition der Hauptfunktion des Produktes in SubFunktionen (ohne Einschränkungen durch ein vorhandenes physisches System).

3. Aufbau der existierenden Produktstruktur durch Identifizieren von Komponenten, Schnittstellen und Baugruppen des Produktes (sofern ein vorhandenes Produkt modularisiert werden soll; für Neuproduktentwicklungen wird dieser Schritt ggf. anders ausgeführt).

4. Verknüpfung von Subfunktionen auf Komponenten und anschließend bilden neuer Module, sodass eine Gruppierung von Komponenten anhand ihres funktionalen Zusammenwirkens erfolgt.

Das resultierende Produktarchitekturkonzept wird anschließend hinsichtlich Schnittstellenoptimierung, „Make-or-Buy“-Analysen sowie einer Verbesserung der Lieferantenstruktur bewertet. Insgesamt besteht die METUS Methodik aus 18 sogenannten Lösungsbausteinen, die eine generische Sequenz bilden, anhand derer Produktentwickler durch den Modularisierungsprozess geführt werden.

Der METUS-Ansatz ist in eine gleichnamige Software implementiert, um eine hohe Anwendungsfreundlichkeit sowie eine einfache Bearbeitung und Handhabung von Daten zu unterstützen. Damit können unterschiedliche Produktanwendungsfälle modelliert sowie komplexe Produktstrukturen und Abhängigkeiten transparent abgebildet werden. Darüber hinaus fördert die Software die Zusammenarbeit interdisziplinärer Experten. Dies erlaubt die Konsolidierung des entsprechenden Expertenwissens in ein Datenmodell, das als kumulierter Wissensspeicher fungiert und bei hinreichender Pflege den Anwender zum Ableiten von semantischen Regeln befähigt.

\subsubsection{Anwendung der Modularisierungsabfolge auf ein Supply Chain Management-Projekt}

In diesem Abschnitt werden die vier Modularisierungsschritte gemäß Göpfert and Tretow (2013) auf das Beispiel eines SCM-Projektes angewendet, um die Vision und das Potential eines MPPM-Ansatzes zu verdeutlichen. 


\subsubsection{Schritt 1: Analyse von Zielen und Anforderungen}

Ziele der Modularisierung wie auch die Anforderungen an ein SCM-Projekt können vielfältig sein. Neben Zielen, die aus einem betriebswirtschaftlichen Hintergrund resultieren wie beispielsweise Kosten-, Zeit-, Qualität-, Flexibilitätund Risiko-Kriterien (Kersten et al., 2012), sind klassische Anforderungen der Logistik zu erfüllen, die beispielsweise auf die Beschaffenheit eines $\mathrm{zu}$ transportierenden Gutes zurückzuführen sind. So ist denkbar, dass während eines Transports die Kühlkette nicht unterbrochen werden darf. Zudem müssen möglicherweise spezifische Zollbestimmung als Anforderung berücksichtigt werden. Im ersten Schritt der Modularisierungsabfolge werden solche Ziele und Anforderungen für das Supply Chain Projekt definiert (vgl. Abbildung 3), um Randbedingungen für den Modularisierungsprozess festzulegen und etwaige Modultreiber abzuleiten.

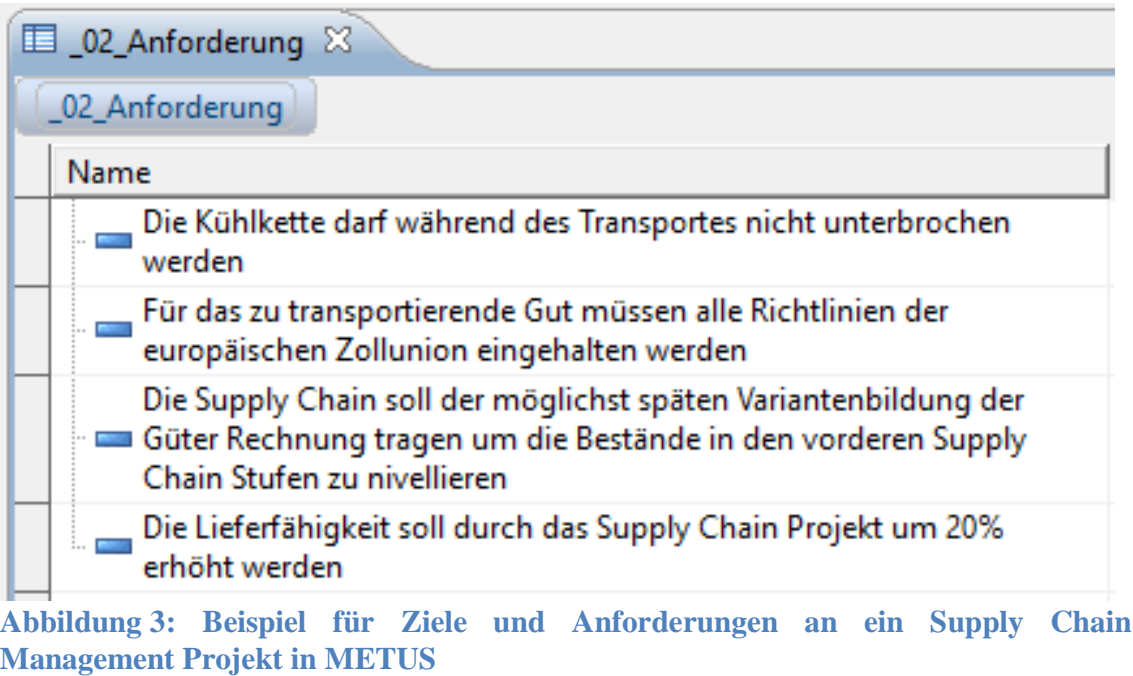

\subsubsection{Schritt 2: Aufbau einer lösungsneutralen Funktionsstruktur}

Beim Aufbau einer Funktionsstruktur wird die Gesamtfunktion eines SCMProjektes (hier „Ebene 1“) auf einer untergeordneten Ebene (Ebene 2) in einzelne Teilfunktionen heruntergebrochen (vgl. Abbildung 4). In der Praxis wird typischerweise noch eine dritte Ebene aufgespannt, worauf in dieser 
exemplarischen Ausführung zum Beibehalten der Einfachheit verzichtet werden soll. Jede der Ebenen der Funktionsstruktur soll eine vollständige und lösungsneutrale Beschreibung der Projektfunktionen darstellen, allerdings jeweils in einem unterschiedlichen Granularitätsgrad. Lösungsneutral bedeutet in diesem Kontext, dass beispielsweise das Transportieren von Gütern mit unterschiedlichen Transportmodi ausgeführt werden kann, dies aber in dieser funktionalen Perspektive nicht determiniert und festgelegt werden soll, damit auch neue und innovative Lösungsansätze einbezogen werden können.

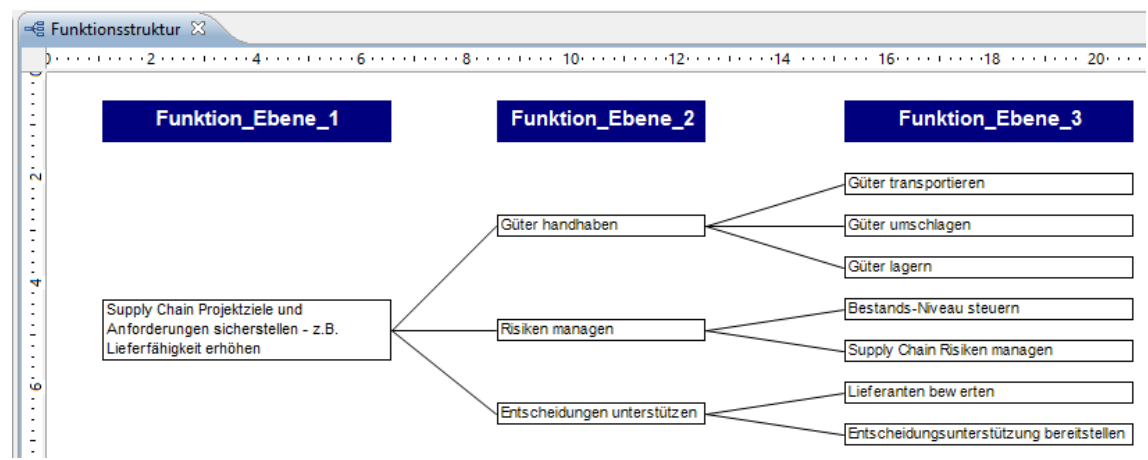

Abbildung 4: Modellierung der lösungsneutralen Funktionsstruktur in METUS

\subsubsection{Schritt 3: Aufbau der existierenden Projektstruktur}

In einem SCM-Projekt wird die Projektstruktur aufgebaut - analog zur Produktstruktur bei der Modularisierung eines Produktes. Eine solche Projektstruktur ist als Beispiel in der rechten Hälfte von Abbildung 5 aufgetragen.

Von der hierarchischen Einordnung in die MPPM-Struktur handelt es sich bei diesen um „Arbeitspakete“. Auf der darunterliegenden Tätigkeitsebene befinden sich in diesem Fall „Supply Chain Tasks“. Am Beispiel des Transports können diese unterschiedliche Transportmodi darstellen, wie bspw. Straße, Schiene, Seefracht und Luftfracht mit jeweils unterschiedlichen Eigenschaften (Abbildung 5).

Wird die Betrachtungsperspektive von Produkten auf ein SCM-Projekt verändert, lassen sich die Arbeitspakete zum Umschlag als „Schnittstellen“ innerhalb einer Prozessabfolge charakterisieren. Weitere Schnittstellenarten in 
diesem Kontext können neben Materialflüssen auch Informations- sowie monetäre Flüsse sein (Lammers, 2012, p. 93).

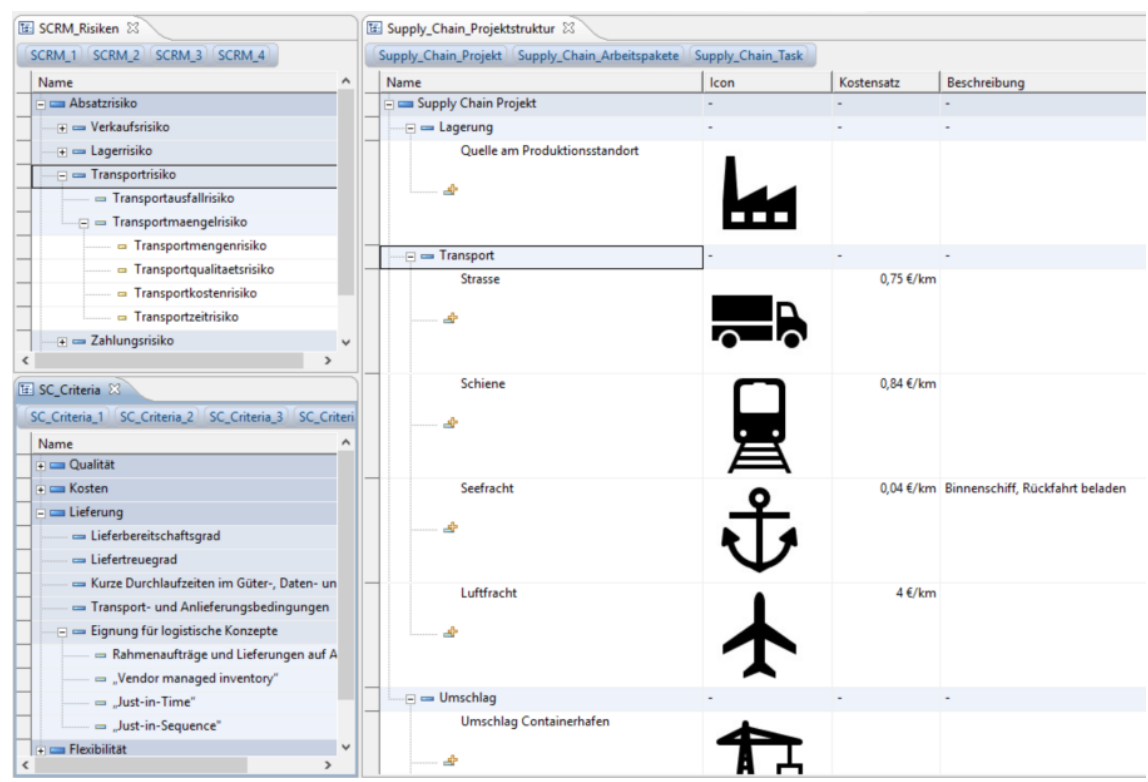

Abbildung 5: Supply Chain Management Projektstruktur in METUS

\subsubsection{Schritt 4: Verknüpfung von Funktionsstruktur und Projektstruktur}

Im Falle des betrachteten SCM-Projekts werden die Elemente der Projektstruktur auf der untersten Tätigkeitsebene als „Supply Chain Tasks“ bezeichnet. Werden diese mit der lösungsneutralen Funktionsstruktur verknüpft, so erhält man die generische Projektarchitektur. Die Verknüpfung kann mit Hilfe der METUSSoftware durchgeführt und visualisiert werden (vgl. Abbildung 6).

Anschließend können die ursprünglich funktional definierten Arbeitspakete flexibel per „drag-and-drop“ in ihrem Umfang verändert und angepasst werden. Das Clustern bzw. Bündeln von verschiedenen „Tasks“ ermöglicht auf diese Weise die Ableitung von Arbeitspaketen unterschiedlichen Umfangs abhängig von den jeweils relevanten Modultreibern. Beispielsweise kann ein Arbeitspaket so zugeschnitten werden, dass dessen Realisierung im Projektkontext für die externe Vergabe an einen Dienstleister in Frage kommt. Ebenso ist aber auch das 
Schneiden von Modulen zur Optimierung von betriebswirtschaftlichen Größen wie Durchlaufzeit oder Kosten eine mögliche Zielsetzung.

Darüber hinaus lassen sich weitere Perspektiven aus dem Datenmodell in die Visualisierung der End-to-End Datenkette einbinden. In der Abbildung werden beispielsweise die übergeordneten Anforderungen an das Projekt mit zu erfüllenden Projektfunktionen verknüpft. 


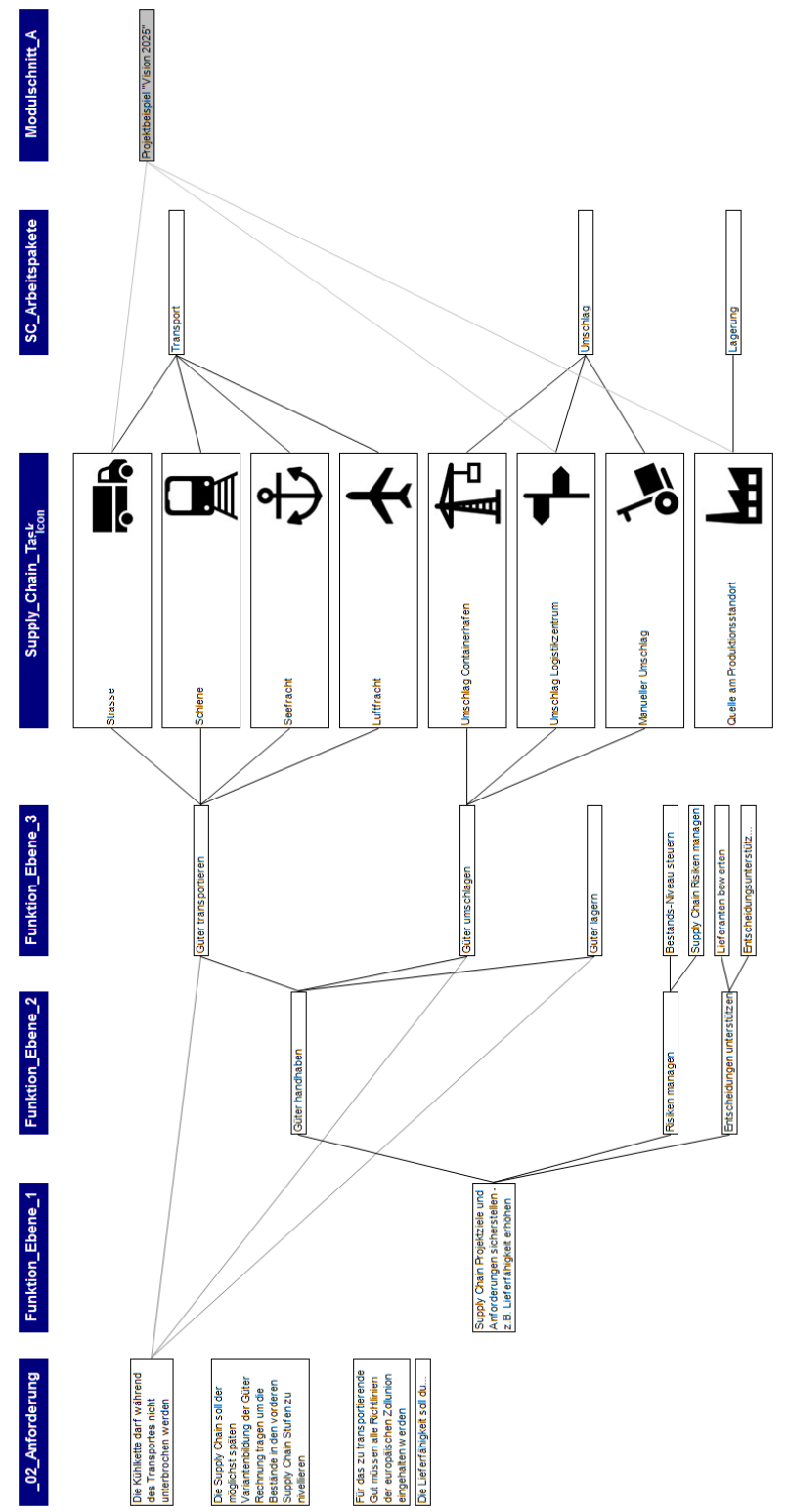

Abbildung 6: End-to-End Datenhandling des SCM-Projektes in METUS 


\subsection{Diskussion und Ausblick}

\subsubsection{Prüfung der Anforderungserfüllung für SCM Projekte}

Nachdem die Anwendung des METUS-Modularisierungsansatzes auf ein SCMProjekt gezeigt wurde, soll nachfolgend diskutiert werden, inwieweit die in Tabelle 2 aufgezeigten Anforderungen an einen agilen Projektportfoliomanagementansatz damit prinzipiell erfüllt werden:

- Die Anpassbarkeit an verschiedene Anwendungskontexte kann als erfüllt angesehen werden, da ein Übertragen auf das Beispiel eines SCM Projektes in diesem Beitrag gezeigt werden konnte.

- Die effiziente Adaptierbarkeit der Projektlandschaft an interne und externe Veränderungen kann durch das Modellieren in der Software aufwandsarm durchgeführt werden.

- Eine Berücksichtigung verschiedener Projektbewertungskriterien wird in der der aufgezeigten Beispielmodellierung in die Bausteine der Projektstruktur integriert und kann flexibel erweitert werden.

- Insgesamt liegt durch den METUS Ansatz ein strukturiertes und systematisches Vorgehen vor, dass zur Qualität der erzielten Ergebnisse beiträgt. Zudem können durch die flexible Modellierung Organisationsstrukturen und Prozesse für das SCM Projekt berücksichtigt werden.

- Das vorgestellte Beispiel fördert die Transparenz. Zum einen können damit einzelne Projektaktivitäten berücksichtigt und erfasst werden. Zum anderen kann durch den umfassenden Projektüberblick die Transparenz von Entscheidungsprozessen gefördert werden.

Durch den begrenzten Umfang und die theoretische Natur des vorgestellten SCM-Beispiels handelt es sich hierbei um eine initiale Bewertung. Im Sinne einer agilen Vorgehensweise wird ein durchgängiges Projektdatenmodell Schritt für Schritt und in der Regel top-down aufgebaut und modelliert.

Durch die modulare und adaptierbare Struktur des METUS-Ansatzes und Software ist eine einfache Adaption von produktspezifischen auf andere Anwendungsfällen möglich, wie hier anhand des Supply Chain ManagementProjekts gezeigt.

Diese grundsätzliche Möglichkeit, die Betrachtung eines Produktes durch die Betrachtung eines Projektes zu ersetzten, wie in diesem Kapitel am Beispiel des Supply Chain Managements erläutert wurde, macht METUS zu einem vielversprechenden Kandidaten für die Umsetzung der Vision eines MPPM- 
Ansatzes. Innerhalb der generischen Projektarchitektur lassen sich anschließend die verschiedenen Instanzen einzelner Projekte handhaben, die in Summe das Projektportfolio darstellen.

Die Möglichkeit, vorhandene generische Modulbausteine für ein SCM-Projekt wiederzuverwenden, verspricht einen hohen Effizienzgewinn. Zudem kann durch die Integration von bereits vorliegenden Erfahrungen in solchen Modulbausteinen die Robustheit bei der Anwendung gesteigert werden.

\subsubsection{Mögliche Erweiterungen als nächste Schritte}

Basierend auf dem beschriebenen modularen SCM-Projekt liegen vier Erweiterungen nahe - beispielsweise durch neue ergänzende Arbeitspakete für Projekte innerhalb des Projektportfolios:

- Erstens scheinen Ergänzungen um Supply Chain-Risiken vielversprechend, die einzelnen Transportarten durch Verknüpfung der Datenelemente aufwandsarm zugeordnet werden können.

- Zweitens liegt eine differenzierte Lieferantenbewertung anhand von mehreren Kriterien nahe, die in den End-to-end Datenhaltungsprozess eingebettet werden kann. Dies kann bis hin zur Bewertung alternativer Sourcing Szenarien über mehrere Supply Chain-Stufen ausgebaut werden.

- Drittens kann über eine Betrachtung hinsichtlich „Modular Sourcing“ die Verknüpfung eines physischen Produktes mit der prozessualen Perspektive in der Supply Chain vorgenommen werden. Optimierungspotentiale resultieren hier beispielsweise durch späte Variantenbildung und Bestände-Pooling innerhalb der gesamten Supply Chain.

- Viertens kann durch das Einbeziehen von Bewertungskriterien für das Supply Chain Management und deren anforderungsbasierte Gewichtung ein Management-taugliches Instrument zur Steuerung und Entscheidungsunterstützung innerhalb eines Projektes, aber auch übergreifend für mehrere Projekte innerhalb eines modularen Projektportfolios zur Verfügung gestellt werden.

Darüber hinaus scheinen drei Erweiterungen zielführend, die aus dem Kontext des Modularen Projektportfolio Managements (MPPM) entstammen:

- $\quad$ Eine Erweiterung des Betrachtungsumfanges eines einzelnen Projektes auf mehrere Projekte, die sich innerhalb eines Projektportfolios befinden und über gleiche Bausteine verfügen. 
- Zudem kann ein höherer Detaillierungsgrad der Modellierung in der METUS Software dazu führen, dass die Unterstützung von Entscheidungen verbessert wird.

- Letztlich würde ein produktiver Einsatz des METUS Tools in der Praxis erfordern, dass geeignete Daten-Schnittstellen $\mathrm{zu}$ vorhanden Projektmanagement-Tools etabliert werden, damit der Datenaustausch möglichst einfach und automatisiert vorgenommen werden kann. 


\section{Zusammenfassung und Ausblick}

In den letzten Jahren hat sich ,agil“" zu einem disziplinübergreifenden Trendwort entwickelt, verbunden mit einer zunehmenden Verbreitung ,agiler Ansätze“. Dennoch lässt sich eine immer noch existierende Uneindeutigkeit beobachten, was dies genau im Kontext von Projektmanagement bedeutet und wie es effizient gemanagt werden kann - speziell im Rahmen von Projektportfolios mit mehreren verknüpften Projekten. Obwohl klassisches Projektportfoliomanagement gerade hier seine Stärken beim strategischen Management mehrerer Projekte hat, weist es essentielle Schwächen hinsichtlich operativem und schnellen flexiblem Projektmanagement auf.

Dieser Beitrag schlägt einen neuen Ansatz vor, wie sich Agilität innerhalb des Projektportfoliomanagement durch die Adaption von Produktmodularisierungsansätzen realisieren lässt. Wie im Beitrag diskutiert, besitzen Projekt- und Produktportfolien eine hohe strukturelle Ähnlichkeit, die es erlaubt Ansätze zwischen beiden Domänen zu transferieren. Aus der Literatur abgeleitete Anforderungen beschreiben, wie Modularisierungsansätze für eine Anwendung im Projektportfoliomanagement entsprechend angepasst werden müssen. Basierend hierauf wird anhand eines exemplarischen Supply-ChainProjektportfolios skizziert, wie die Vision eines „Modularen Projektportfoliomanagements (MPPM)“ aussehen könnte. Auch wenn es sich hierbei bislang nur um konzeptionelle Ergebnisse handelt, sind diese sehr vielversprechend und motivieren $\mathrm{zu}$ einer detaillierten forschungstechnischen Betrachtung.

Während der hierbei resultierende wissenschaftliche Mehrwert unter anderem in einem Beitrag zum Themenfeld Agilität und ihres Managements sowie zu einem disziplinübergreifenden Wissenstransfers liegt, ergäben sich auch für die Praxis greifbare Vorteile im Bereich des Projektportfoliomanagements. Neben einem flexiblen Management und einem schnelleren Reagieren auf un-/vorhergesehene Ereignisse, erlaubt ein MPPMAnsatz die Erstellung von Projektbaukästen mit standardisierten Projektbausteinen für eine schnelle und einheitliche Projektplanung in Unternehmen sowie den Aufbau eines kumulierten Wissensspeichers für Lessons Learnt aus der Anwendung dieser Bausteine. Die Berücksichtigung verschiedener Modultreiber und ein resultierender projektspezifischer Modulbzw. Arbeitspaketschnitt reduziert Abhängigkeiten zwischen Projektelementen und mögliche negative Projektwechselwirkungen, falls sich ein Projekt negativ entwickelt. Klar definierte Zwischenergebnisse jedes Arbeitspakets unterstützen das Projektcontrolling sowie die Wiederverwertbarkeit und projektübergreifende Nutzung von Arbeitsergebnissen. 


\section{Zitierhinweise:}

Archer, N.P., Ghasemzadeh, F., 1999. An integrated framework for project portfolio selection, International Journal of Project Management, vol. 17, no. 4, pp. 207-216.

Bask, A., Lipponen, M., Rajahonka, M., Tinnilä, M., 2011. Modularity in logistics services: A business model and process view, International Journal of Services and Operations Management, vol. 10, no. 4, p. 379.

Behnke, F., 2016. Beschaffungsgerechte Produktentwicklung - Abstimmung von Produktarchitektur und Liefernetzwerk in frühen Phasen der Entwicklung, Dissertation, München, Technische Universität München.

Blees, C., Jonas, H., Krause, D., 2010. Development of modular product families, DRUID-DIME Academy Winter Conference 2010: Innovation, Knowledge and Entrepreneurship. Aalborg, Denmark, January 21-23 2010.

Blichfeldt, B. S., Eskerod, P., 2008. Project portfolio management - There's more to it than what management enacts, International Journal of Project Management, vol. 26, no. 4, pp. 357-365.

Bremner, R., 1999. Cutting edge platforms. Financial Times Automotive World, Financial Times Automotive World, September, pp. 30-38.

Bruno, J., 2005. Projektmanagement. Das Wissen für eine erfolgreiche Karriere, 2nd edn, Vdf Hochschulverlag, Zürich.

Darrin, M. A. G., Devereux, W. S., 2017. The Agile Manifesto, design thinking and systems engineering, 2017 Annual IEEE International Systems Conference (SysCon). Montreal, QC, Canada, 24/04/2017 - 27/04/2017, IEEE, pp. 1-5.

Golfmann, J., Lammers, T., 2015. Modular Product Design: reducing complexity, increasing efficacy, Performance Journal, EY, vol. 7, no. 1, pp. 56-63.

Göpfert, J., 2009. Modulare Produktentwicklung. Zur gemeinsamen Gestaltung von Technik und Organisation ; Theorie, Methodik, Gestaltung, 2nd edn, Books on Demand, Norderstedt.

Göpfert, J., Tretow, G., 2013. Produktarchitektur, in Feldhusen, J. and Grote, K. H. (eds) Pahl/Beitz Konstruktionslehre: Methoden und Anwendung erfolgreicher Produktentwicklung, 8th edn, Berlin, Heidelberg, Imprint: Springer Vieweg, pp. 252-278.

Haskins, C., 2006. Systems Engineering handbook, International Council on Systems Engineering (INCOSE).

Herberg, A., 2017. Planung und Entwicklung multifunktionaler Kernmodule in komplexen Systemarchitekturen und -portfolios. Methodik zur Einnahme 
einer konsequent modulzentrierten Perspektive, Dissertation, Technische Universität München.

Kaiser, M. G., El Arbi, F., Ahlemann, F., 2015. Successful project portfolio management beyond project selection techniques: Understanding the role of structural alignment, International Journal of Project Management, vol. 33, no. 1, pp. 126-139.

Kersten, W., Henning Skirde, Lammers, T., 2013. Komplexitätsmanagement durch Modular Sourcing - die Rolle von Patenten bei unternehmensübergreifenden Modulstrategien, in Friedewald, A. and Lödding, H. (eds) Produzieren in Deutschland - Wettbewerbsfähigkeit im 21. Jahrhundert, Berlin, Gito Verlag, pp. 221-238.

Kersten, W., Hülle, J., Möller, K., Lammers, T., 2009. Kostenorientierte Analyse der Modularisierung, ZWF Zeitschrift für wirtschaftlichen Fabrikbetrieb, vol. 104, no. 12, pp. 1136-1141.

Kersten, W., Möller, K., Sedlmeier, L., Skirde, H., 2012. Analyzing the Cost Effects of Modularity - Requirements for the Development of a Methodology, in Kersten, W. (ed) Managing the Future Supply Chain: Current concepts and solutions for reliability and robustness, Lohmar, Eul, pp. $153-165$.

Killen, C. P., Kjaer, C., 2012. Understanding project interdependencies: The role of visual representation, culture and process, International Journal of Project Management, vol. 30, no. 5, pp. 554-566.

Koppenhagen, F., 2004. Systematische Ableitung modularer Produktarchitekturen. Komplexitätsreduzierung in der Konzeptphase, Shaker, Aachen.

Lammers, T., 2012. Komplexitätsmanagement für Distributionssysteme. Konzeption eines strategischen Ansatzes zur Komplexitätsbewertung und Ableitung von Gestaltungsempfehlungen (Zugl.: Hamburg-Harburg, Techn. Univ., Inst. für Logistik und Unternehmensführung, Diss., 2012), Eul, Lohmar.

Levine, H. A., 2005. Project portfolio management. A practical guide to selecting projects, managing portfolios, and maximizing benefits / Harvey A. Levine ; foreword by Max Wideman, Jossey-Bass; Chichester : John Wiley [distributor], San Francisco, Calif.

Lindemann, U., Maurer, M., Braun, T., 2009. Structural Complexity Management, Springer, Berlin, Heidelberg.

Lycett, M., Rassau, A., Danson, J., 2004. Programme management: A critical review, International Journal of Project Management, vol. 22, no. 4, pp. 289 299. 
Mack, O., Khare, A., Krämer, A., Burgartz, T., 2016. Managing in a VUCA World, Springer International Publishing, Cham.

Martinsuo, M., 2013. Project portfolio management in practice and in context, International Journal of Project Management, vol. 31, no. 6, pp. 794-803.

Martinsuo, M., Killen, C. P., 2014. Value Management in Project Portfolios: Identifying and Assessing Strategic Value, Project Management Journal, vol. 45 , no. 5 , pp. 56-70.

Martinsuo, M., Korhonen, T., Laine, T., 2014. Identifying, framing and managing uncertainties in project portfolios, International Journal of Project Management, vol. 32, no. 5, pp. 732-746.

Mayer, A., 2007. Modularisierung der Logistik. Ein Gestaltungsmodell zum Management von Komplexität in der industriellen Logistik (Zugl.: Berlin, Techn. Univ., Diss., 2007), Univ.-Verl. der Techn. Univ, Berlin.

Parvan, K., Rahmandad, H., Haghani, A., 2015. Inter-phase feedbacks in construction projects, Journal of Operations Management, 39-40, pp. 48-62.

Patanakul, P., 2015. Key attributes of effectiveness in managing project portfolio, International Journal of Project Management, vol. 33, no. 5, pp. 1084-1097.

Pekkarinen, S., Ulkuniemi, P., 2008. Modularity in developing business services by platform approach, The International Journal of Logistics Management, vol. 19 , no. 1, pp. 84-103.

Persson, M., Åhlström, P., 2013. Product modularisation and organisational coordination, International Journal of Automotive Technology and Management, vol. 13, no. 1, p. 55.

Reyck, B. D., Grushka-Cockayne, Y., Lockett, M., Calderini, S. R., Moura, M., Sloper, A., 2005. The impact of project portfolio management on information technology projects, International Journal of Project Management, vol. 23, no. 7, pp. 524-537.

Rungi, M., 2010. Success rate and resource consumption from project interdependencies, Industr Mngmnt \& Data Systems, vol. 110, no. 1, pp. 93110.

Sarkar, A., 2016. We live in a VUCA World: The importance of responsible leadership, Development and Learning in Organizations, vol. 30, no. 3, pp. 9-12.

Schrieverhoff, P., 2015. Valuation of Adaptability in System Architecture, Dissertation an der Technischen Universität München, München.

Schuh, G., 2005. Produktkomplexität managen. Strategien, Methoden, Tools, 2nd edn, Hanser, München.

Schuh, G., Arnoscht, J., Vogels, T., 2013. Controlling der Varianzsensitivität in Baukastensystemen, Controlling, vol. 25, no. 2, pp. 82-89. 
Sedlacek, K.-D., 2010. Emergenz. Strukturen der Selbstorganisation in Natur und Technik : Arbeits- und Handbuch, Books on Demand, Norderstedt.

Skirde, H., 2015. Kostenorientierte Bewertung modularer Produktarchitekturen (Zugl.: Hamburg-Harburg, Techn. Univ., Institut für Logistik und Unternehmensführung, Diss., 2015) [Online], Eul, Lohmar. Available at http://api.vlb.de/api/v1/asset/mmo/file/01d38f6d-8994-40b3-a407$55833015 \mathrm{ef} 4 \mathrm{e}$.

Skirde, H., Kersten, W., Schröder, M., 2016. Measuring the Cost Effects of Modular Product Architectures - A Conceptual Approach, International Journal of Innovation and Technology Management, vol. 13, no. 04, p. 1650017.

Ulrich, K., 1995. The role of product architecture in the manufacturing firm, Research Policy, vol. 24, no. 3, pp. 419-440.

Unger, B. N., Gemünden, H. G., Aubry, M., 2012. The three roles of a project portfolio management office: Their impact on portfolio management execution and success, International Journal of Project Management, vol. 30, no. 5 , pp. 608-620.

Verma, D., Sinha, K. K., 2002. Toward a theory of project interdependencies in high tech R\&D environments, Journal of Operations Management, vol. 20, no. 5 , pp. 451-468. 